\title{
Kernos
}

Revue internationale et pluridisciplinaire de religion grecque antique

\section{2| 1999}

Varia

\section{A. AVANZINI (éd.), Profumi d'Arabia}

\section{Annick Lallemand}

\section{(2) OpenEdition}

\section{Journals}

Édition électronique

URL : http://journals.openedition.org/kernos/748

DOI : $10.4000 /$ kernos.748

ISSN : 2034-7871

Éditeur

Centre international d'étude de la religion grecque antique

Édition imprimée

Date de publication : 1 janvier 1999

Pagination : 324-326

ISSN : 0776-3824

Référence électronique

Annick Lallemand, «A. Avanzinı (éd.), Profumi d'Arabia », Kernos [En ligne], 12 | 1999, mis en ligne le 13 avril 2011, consulté le 11 mars 2021. URL : http://journals.openedition.org/kernos/748 ; DOI : https:// doi.org/10.4000/kernos.748 
Alessandra Avanzini (a cura di), Profumi d'Arabia. Atti del Convegno, Roma, "L'Erma" di Bretschneider, 1997. 1 vol. $14 \times 20,5 \mathrm{~cm}, 564$ p., ill. (Saggi di Storia Antica, 11). ISBN : 88-7062-975-9

La collection «Saggi di Storia Antica » nous offre de nouveau une contribution importante à la connaissance des aromates dans l'Antiquité; après la parution du volume 9, Cassia, Cinnamomo, Ossidiana de F. De Romanis en 1996, voici le volume 11, épais ouvrage collectif consacré aux productions de l'Arabie, encens et myrrhe. Il s'agit des actes d'un colloque qui s'est tenu à Pise, faisant suite au colloque de Strasbourg de 1987 (Actes publiés en 1989, L'Arabie préislamique et son environnement bistorique, éd. T. Fahd); A. Avanzini, elle-même très au fait du sujet, a réuni les meilleurs spécialistes de l'Arabie antique et publié 38 communications d'une telle densité qu'il est impossible d'en rendre compte dans le détail; je signalerai surtout celles qui concernent le domaine grec, même si l'essentiel du livre n'est pas là.

Ce colloque a essayé de dépasser le stéréotype littéraire de l'Arabie heureuse et de percer le mystère de ses aromates à la lumière des plus récentes découvertes archéologiques; il a mis en évidence l'intérêt tout spécial porté par les archéologues italiens à la Péninsule arabe et a souligné le dynamisme de la recherche internationale dans ce domaine. En France, le colloque de Pise a été suivi de deux très belles expositions qui ont mis un large public au courant des dernières découvertes, «Yémen. Au pays de la reine de Saba » à l'Institut du monde arabe à Paris, "Voyage au Pays de Pount » à Aix en Provence; nous sommes très redevables de la qualité de ces expositions à Ch. Robin, auteur d'un article récent fondamental "Sheba. II. Dans les inscriptions d'Arabie du Sud " (Supplément au Dict. de la Bible, fascicule 70, Paris, 1996, 1047-1254), et présent à Pise avec une communication très savante et très claire sur « Arabie méridionale : l'état et les aromates » (p. 37-56).

Les articles (18 en anglais, 12 en italien, 4 en français, 1 en allemand), sont regroupés en cinq parties, sans compter l'introduction et la conclusion: les origines, les objets (que l'on trouve forcément aussi dans les autres parties), les mots, les routes, l'imaginaire :

- les origines (p. 23-84): A. Avanzini avait rappelé dans l'introduction qu'un des apports récents les plus importants est sans doute la chronologie; le début de la documentation écrite en Arabie peut être daté du premier millénaire (et non plus du milieu du premier millénaire), époque du développement du commerce des aromates; mais les liens avec la Mésopotamie sont étudiés depuis le $3^{\mathrm{e}}$ millénaire dans toute leur complexité (S. Mazzoni); une nouvelle technique de construction de bateaux en bois est attestée en Oman vers 2300 av. J.-C. (étude magistrale de $S$. Cleuziou - M. Tosi).

- les objets (p. 85-168): on apprendra beaucoup sur les différents types d'encensoirs appelés aussi pyrées, brûle-parfums, autels à parfums... en métal ou en pierre dans l'Orient classique en lisant l'article richement documenté d'A. Invernizzi qui domine deux millénaires; en Sibérie notamment ont été trouvés des objets dans un état de conservation exceptionnel. En comparaison, l'article précédent sur «l'encens et les encensoirs en Grèce » rle C. Zaccagnino fait un peu pâle figure, sans doute à l'image de la réalité grecque; selon l'auteur, tous les dieux grecs recevaient des offrandes d'encens; sont analysés les cas d'Apollon et d'Héra; Aphrodite est seulement citée sans exemple à l'appui. Cet essai déjà intéressant mériterait d'être poussé notamment avec une étude plus approfondie de la représentation des encensoirs sur les vases. 
- les mots (p. 169-250) : des articles, tous aussi savants (G. Banti - R. Contini, W.W. Müller, J. Ryckmans), décryptant les noms des aromates dans les langues sémitiques et cushitiques, les inscriptions éthiopiennes, sudarabiques, les textes minéens, égyptiens... feront la joie des linguistes. F. De Romanis a tenté de mettre en relation les emprunts et les évolutions avec les usages dans la société (tus et murra dans la Rome archaïque). Le lecteur profane appréciera une mise au point botanique sur l'arbre à encens lui-même Boswellia sacra (M. Morris, à propos de la façon traditionnelle de récolter la résine).

- les routes (la partie la plus importante, p. 251-448): des études très fines complètent ou rectifient les tracés présentés dans le livre de N. Groom, Frankincense and Myrrb. A Study of the Arabian Incense Trade (Londres, 1981) car, comme le fait remarquer A. De Maigret, "d'une manière très ironique, les routes caravanières de l'encens sont à peine mentionnées dans les textes d'Arabie du Sud ». Sont donc mis en évidence les contacts de l'Arabie du Sud avec la Mésopotamie depuis le néolithique, 3200 av. J.-C. (J. Zarins, article remarquable comportant une étude de l'écologie du Dhofar et une bibliographie très complète), avec la Corne de l'Afrique depuis la fin de l'époque préhistorique (R. Fattovich); sont étudiés ses échanges avec la péninsule d'Oman depuis le $\operatorname{III}^{\mathrm{e}} \mathrm{s}$. av, J.-C. (M. Mouton), avec l'Égypte ptolémaïque (U. Fantasia), le point d'aboutissement septentrional de la route de l'encens (M.C.A. Macdonald, qui utilise parfaitement les données bibliques). L'étude de T. Gnoli sur le baume de Judée dans l'oasis d'Engaddi est très originale. Le lecteur chemine donc avec hésitation à dos d'âne jusqu'au premier millénaire; puis, à dos de chameau ou de dromadaire, il peut parcourir $2560 \mathrm{~km}$ de Najran à Gaza en 62-66 jours (moyenne par jour 41,6 km selon A. De Maigret, p. 324). Les routes maritimes, peut-être plus anciennes, sont également scrutées (J. Owen, A.V. Sedov). Enfin P.M. Costa nous emmène du côté de l'Océan indien, de 24 ap. J.-C. jusqu'à l'époque de Vasco de Gama. Des cartes accompagnent la plupart des exposés; celle de la p. 394 du désert oriental d'Égypte est un modèle de clarté.

- l'imaginaire (p. 449-542) : cette partie m'a paru la plus inégale, notamment en ce qui concerne la Grèce. Les liens entre aromates et royauté sont montrés dans toute leur complexité à propos d'Antiochos IV (C. Grottanelli); la synthèse de $G$. Salmeri sur l'usage de l'encens à l'époque tomaine est tout à fait bienvenue. J. Retsö pose la question des implications politiques de l'encens entre 460 et 350 av. J.-C. et du rôle de Chypre sans arriver à une réponse convaincante. L'article verbeux d'A. Zambrini, "Notes sur l'Arabie dans les sources classiques", n'est pas à sa place dans un recueil de ce niveau. L. Soverini, de manière vivante, commente le passage très connu du Banquet de Xénophon où Socrate refuse l'usage des parfums dans les banquets, en l'opposant à un goût supposé des sophistes pour les parfums; j'avais montré dans un article, "Flâner sur le marché aux parfums » (Les loisirs et l'béritage de la culture antique, Bruxelles, 1996, p. 76-84), comment les parfums orientaux étaient devenus à la mode à Athènes à l'époque classique; la réaction ascétique de Socrate est tout à fait attendue, et "l'odeur de la vertu » promise à une grande destinée.

Il m'a semblé un peu dangereux, dans un colloque d'historiens, de mélanger les notions d'aromates et de parfums. Sans doute le titre «Parfums d'Arabie » sonne-t-il mieux qu' « Aromates d'Arabie »; mais l'Arabie n'a pas fabriqué des parfums, elle a produit et exporté des résines aromatiques; et les auteurs, quand ils ont joué sur les mots, n'ont pas abouti à un résultat tout à fait satisfaisant. Dans la conclusion, G.W. Bowersock, à propos du lien entre souveraineté et encens, affirme sans nuance p. 555 que « les auteurs de l'Antiquité tardive et de Byzance de façon notoire ne portèrent aucun intérêt aux parfums »; le témoignage, 
même réprobateur, de Jean Chrysostome suffit à le contredire : à Constantinople les femmes chrétiennes raffolent des parfums, et même le moine, qui vit avec une vierge, " court chez le parfumeur pour s'entretenir avec lui des aromates de madame »(Les cohabitations suspectes, 10). Par contre, manquent dans ce colloque des communications sur l'encens dans l'imaginaire juif et l'imaginaire chrétien antiques; j'avais souligné la continuité de la représentation de l'Arabie, de merveille géographique en pays divin, jusque chez les auteurs chrétiens, dans un article « Le parfum comme signe fabuleux dans les pays mythiques » (Peuples et pays mythiques, Paris, 1988, p. 73-90). Le choix d'un détail d'une peinture italienne représentant Marie-Madeleine pour la jaquette traduit ce manque; un objet typique de l'Arabie du Sud aurait été plus représentatif du contenu véritable du livre.

En somme cet ouvrage fera référence, et sera un guide aussi sûr que passionnant pour le lecteur qui veut s'aventurer sur la route de l'encens.

Annick Lallemand

(Paris IV - Sorbonne)

María José Hidalgo de la Vega, Juan José Sayas Abengochea \& José Manuel Roldán Hervás, Historia de la Grecia Antigua, Salamanca, Ediciones Universidad, 1998. 1 vol. $17 \times 24,5 \mathrm{~cm}, 485$ p., 18 ill. (Historia Salamanca de la Antigïedad. Manuales universitarios, 58). ISBN : 84-7481-889-3.

Confrontés à la rareté des synthèses d'histoire grecque conçues en Espagne, où une large part revient traditionnellement aux traductions de livres étrangers, les trois auteurs ont uni leurs compétences afin d'élaborer un manuel destiné prioritairement aux étudiants qui abordent pour la première fois l'étude du monde hellénique. María José Hidalgo de la Vega, spécialiste de la religion et du mythe, s'est attachée aux deux premières sections consacrées à la civilisation égéenne ( 3 chapitres) et à la Grèce archaïque ( 8 chapitres). De formation classique, auteur d'une thèse sur la pensée politique de Thucydide, Juan José Sayas Abengochea a rédigé la partie relative à la Grèce classique (8 chapitres). José Manuel Roldán Hervás enfin, qui a surtout étudié les deux derniers siècles de la république romaine, s'est chargé du monde hellénistique (10 chapitres). Par delà la spécificité de chacun, c'est dans un même état d'esprit que les auteurs ont travaillé : depuis l'Âge du Bronze jusqu'à la fin de l'époque hellénistique au $\mathrm{I}^{\text {er }}$ siècle av. J.-C., le monde grec est ici présenté d'une manière qui fait la part belle à la richesse et à la diversité de son histoire et de sa civilisation, avec à l'occasion un écho aux opinions des historiens modernes ce qui permet de ne pas en rester à une présentation unilatérale ou consensuelle. Le tout est exposé de façon approfondie dans une suite de courts paragraphes, avec de nombreux sous-titres, qui soutiennent bien l'attention. Le plus souvent, les chapitres se terminent par une chronologie et une orientation bibliographique autour des principaux thèmes qui viennent d'être développés, la bibliographie générale étant de son côté assez «symbolique », une vingtaine de titres sans commentaire, dont l'usage n'apparaît guère. Si l'étendue de l'horizon ouvert par ce livre se révèle très stimulante, la place réduite réservée aux sources et aux méthodes ainsi qu'aux conditions de notre connaissance ( $c f$. toutefois, p. 35-37 sur le passage du Néolithique au Bronze Ancien, 41 sur l'« arrivée des Grecs » et le Bronze Moyen, 47-49 sur le Minoen Récent, la thalassocratie de Minos et la fin de la civilisation minoenne, 57-60 sur les sources de l'histoire mycénienne, 66-69 sur les thèses relatives à la fin du monde mycénien, 73, 76-80 à propos des siècles dits obscurs, 128-129 sur la Sparte archaïque, 141-145 sur l'Athènes archaïque, 295-296 sur la 